\title{
Mechanistic Learning Goals Enhance Elementary Student Understanding and Enjoyment of Heart Lessons.
}

\author{
Nicole Betz (nicole.betz@yale.edu) \\ Department of Psychology, Yale University \\ New Haven CT, 06520 USA \\ Frank C. Keil (frank.keil@yale.edu) \\ Department of Psychology, Yale University \\ New Haven CT, 06520 USA
}

\begin{abstract}
Biologists, lay adults, and children alike value causal explanations of how biological entities work. Despite this, elementary school science education has historically lacked mechanistic content. In line with recent science education standards, we investigated the effects of mechanistic learning goals on understanding of an in-depth lesson about how the heart works. Children ages 6 to 11 who were given mechanistic learning goals performed better on knowledge assessments of the heart lesson and enjoyed their learning goal more than children who were given a relatively superficial learning goal-to focus on labels. Thus, learning goals orienting children towards mechanistic content during lessons enhance science learning and enjoyment.
\end{abstract}

Keywords: Mechanistic Explanations; Children; Cognitive Development; Education; STEM Learning

\section{Introduction}

"Look at this!" is often used to direct young children's attention towards a focal object. This phrase helps narrow a child's learning focus from a wide range of possibilities to a specific entity. As children develop, we use different strategies to guide their focus, such as learning goals. This study examined the impact of a mechanistic learning goal on a lesson taught to young school children about the heart. We explored the effects of this mechanistic orientation on both learning during the heart lesson and on enjoyment of the goal.

Scientists strive to develop and communicate mechanistic understandings of natural phenomena. These explanations break a phenomenon down into component parts and describe how components interact to produce the phenomenon (Machamer et al., 2000). Biology relies heavily on mechanistic understandings to describe and explain phenomena (Bechtel \& Abrahamsen, 2005).

Children are driven to learn how things work, often seeking causal or mechanistic explanations to satisfy this curiosity. Young children ask questions that probe for causal explanations (e.g., Frazier, Gelman \& Wellman, 2009; Liquin \& Lombrozo, 2020), and are unsatisfied with explanations that lack clear causal or mechanistic content (Mills et al., 2018). This interest persists across elementary school, as evidenced by curiosities expressed by sixth grade students reflecting on science (e.g., "I was wondering how sounds got through the little cords on [headphones] and came out the ends of the buds"; Luce \& Hsi, 2014).
Despite this abundant mechanistic curiosity, adults may not expect elementary school children to understand or learn about mechanisms. For example, adults believed that a video describing a mechanistic explanation of a car engine would suitable for adults, but not children. This belief contrasts success that children had when learning about engine mechanics from that same video (Chuey et al., under review), as well as other evidence demonstrating that children can understand mechanism (e.g., Metz 1985, 1991; Russ et al., 2009). Moreover, adults think that young children should be taught about broad, superficial biological content (e.g., what the names of different types of plants are) rather than about mechanistic explanations (e.g., how the plants work), in part because they believe that mechanisms are difficult for children to learn (Betz, McCarthy \& Keil, under revision).

In line with these lay intuitions, elementary school science education in the United States has historically focused on superficial breadth of content (Schmidt, Wang \& McKnight, 2005), or science vocabulary (e.g., Snow, 2008). The historical focus on science terminology appears to breed rotememorization (e.g., Lock, 1998) and these terms are quickly forgotten (Jones et al., 2004). For these reasons, recent science standards emphasize the importance of teaching indepth content, such as mechanism, in K-12 education (NGSS, 2013). In-depth content is construed here as long, iterative, and comprehensive coverage about one concept.

Teaching mechanistic content may help learners create particularly strong conceptual or mental models of a scientific concept that support understanding and prediction of the physical world (e.g., Craik, 1943; Gentner \& Stevens, 1983; Moray, 1998). Within mental models, individuals represent the structure of a system, whether it is a concrete process or an unobservable, abstract process like the flow of electricity (Gentner \& Gentner, 1982). The most successful mental models should reflect refined, mechanistic understandings that represent interrelated causal relations (de Kleer \& Brown, 1981). As with mechanistic understandings, mental models help guide questions and predictions about how the system will react to changes (Vosniadou, 2002; Vosniadou \& Brewer, 1994), perhaps by mentally simulating what will happen in certain situations (Collins \& Gentner, 1987; Forbus \& Gentner, 1997). For these reasons, mental models - especially causal or mechanistic ones - may aid in conceptual development, information processing, and the revision of scientific understandings (Vosniadou, 2002). 
Indeed, mechanism-focused explanations appear to bolster children's recollection and inferences surrounding mechanistic processes. For example, four- and five-year-olds given mechanistic explanations were better able to recall taught content and generalize their content to novel entities (Kurkel et al., 2021). We therefore expect that a mechanistic focus should help children create a rich mental model of mechanistic content, thereby enhancing learning.

One way to direct focus towards mechanistic content is through explicit learning goals. Indeed, learning goals can help young children achieve externally imposed objectives. For example, preschoolers presented with a novel toy successfully benefitted from a goal to "figure out how it works" (Bonawitz et al., 2011). These and other findings (e.g., Shunk \& Rice, 1989; McNeil \& Alibali, 2000) demonstrate that young children understand learning goals and use the goals to orient and enhance their learning.

\section{Experiment}

Given the prevalence of such superficial teaching of labels in K-12 schools (for a review, see Jones et al., 2018) and the potential benefits of mechanistic thinking (e.g., Russ et al., 2009; Kurkel et al., 2021), we investigated the effects of orientation towards each type of learning (i.e., labels versus mechanisms) through the use of explicit learning goals. Of particular interest was the potential learning benefits of a mechanistic focus compared to a relatively superficial learning goal: a focus on labels.

While teaching labels can be done in an in-depth way, the simple incidental exposure of terminology without context is considered relatively superficial (e.g., Coyne et al., 2009). We contrast this kind of relatively superficial teaching of labels with an in-depth mechanistic explanation of a complex process: how the parts of the heart pump blood. We taught this process through a richly detailed, story-like explanation. The labels serve as additional information embedded in the story, contextualized by their mechanistic role.

We examined the effects of orienting children toward indepth, mechanistic content of a lesson by suggesting explicit learning goals, predicting that mechanistic learning goals could enhance children's learning and enjoyment.

Specifically, we presented children with either a mechanistic learning goal or a labels learning goal prior to watching an educational video about the heart. Both learning goals were developed to be challenging, task-focused and concrete to maximize their efficacy (for a review of effective learning goals, see Locke et al., 1980). We thereafter assessed children's recall of heart mechanism and labels. We predicted that children who were given a mechanistic learning goal would not only perform better on mechanistic questions, but also would perform better on the labeling questions because the increased causal understanding would help bind together labels in a relational representation (Beuhner, 2012), such as a mental model (e.g., Vosniadou, 2002). Such findings would demonstrate that a focus on mechanism broadly benefits learning.
In short, our study focused on the following questions: Does a focus on mechanism enhance science learning? and Do children prefer mechanistic learning goals to labels?

\section{Method}

Participants. One hundred and eighty children from ages $6 ; 0$ through $11 ; 11$ were recruited and run through an online platform for developmental researchers $(\mathrm{N}=172$; Sheskin \& Keil, 2018) or through elementary school after school programs (N=8) from January 2019 through March 2020. Families participating through the online platform received a $\$ 10$ Amazon gift card, while children who participated in after school programs received a toy. Participants were grouped by age, with 60 participants in each of three age groups: 6- to 7-year-olds, 8- to 9-year-olds, and 10- to 11year-olds. In addition to the final sample of 180,11 children were recruited and run through the task, but were omitted from analyses due to disruptive technical issues $(\mathrm{N}=6)$ or because the child opted out of participation $(\mathrm{N}=5)$. These participants were replaced in the sample to obtain the preregistered sample size.

Learning Goal Manipulation. Participants were randomly assigned to one of two conditions which determined which learning goal they received: mechanistic or labels. In both conditions, participants were first given the same brief description of the heart video and instructed "try to pay attention and learn about the heart."

For the mechanistic learning goal, participants were given the following additional instructions: "Your learning goal toady is to remember as much as you can about what all of the parts of the heart do. It is important to remember what all of the parts do because they all have an important job. When they all work together, they can pump blood through the body. After the video, I'm going to tell you about some hearts that aren't working right and ask you to try to help me figure out what part of the hart may need to be fixed. You can use what you learn about what all of the parts of the heart do to answer these questions."

For the labels learning goal, participants were instead given the following additional instructions: "Your learning goal today is to remember as much as you can about what all of the parts of the heart are called. It is important to remember all of the names of the parts of the heart because all of the parts are important to help pump blood through the body, so we should know what they're all called. After the video, I'm going to ask you to tell me the names of the parts of the heart in a drawing like this one. You can use what you learn about what all of the parts of the heart are called to answer these questions."

Participants were also shown an image presented alongside their learning goal (wrench and screwdriver next to heart for mechanism, heart with questions marks pointing to parts for labels). A small version of this image was displayed in the upper left-hand corner of the remainder of the experiment, including the heart video. Children were told that 
the image was there to serve as a reminder of the learning goal during the task.

Educational Heart Video. We created a four-minute detailed video explaining how the heart pumps blood by editing a pre-existing YouTube video

(https://www.youtube.com/watch?v= qmNCJxpsr0). We edited the audio and video to feature child-friendly language describing the flow of blood through each chamber of the heart and lungs, and to discuss the role of the valves in directing blood flow. We provided labels of the chambers, aorta and pulmonary artery within the video (for mechanistic stimuli, see https://youtu.be/hRM3gCHKHcg). The only difference between the heart videos across the two learning goal conditions was the small image in the top left corner of the video associated with their learning goal.

Distractor Task. In order to avoid mental rehearsal of learned content immediately after the video, participants completed a brief distractor task asking them to choose between two options for four simple questions (e.g., Which do you think is more fun: reading books or playing with friends?). The task took one minute to complete.

Test Phase: Labels Questions. The assessment contained four questions assessing knowledge of part labels.

To assess recall of labels of parts of the heart, participants were shown a screenshot of a heart taken from the video with an arrow pointing to one part of the heart (e.g., Right Atrium). Participants then were given three options for what that name of the part is called (e.g., Right Atrium, Left Aorta, Ventricle). For each question, the answer options were associated with a color and participants were instructed to say the color instead of the name itself, to avoid issues with pronunciation abilities. There were three questions assessing recall of labels (i.e., Right Atrium, Aorta, Pulmonary Artery).

To assess representation of the heart structure, as well as ability to map this structure to a label, participants were asked to report how many chambers there are in a heart (two, four or six). Images of dice were used in this question to represent each of numerical responses. Here, the die depicting the number four showed a rudimentary representation of the four-chambered heat structure.

The four labels questions were blocked together, with both the order of questions and order of response options and associated colors counterbalanced across participants.

Test Phase: Mechanistic Questions. The assessment contained four questions assessing knowledge of the mechanisms within the heart.

To assess understanding of how the heart pumps blood, participants were shown a screenshot of a heart taken from the video and told that the heart was "not working right" because "one of the parts of the heart is broken." The researcher then briefly described what the heart was not doing right (e.g., "the blood is flowing through the heart and into the lungs, but then the blood is not coming back into the heart)" and revealed three colored arrows pointing to different parts of the heart (e.g., right atrium, right ventricle, left atrium). Participants were then asked which color arrow points to the part that is not working right. There were three broken heart questions: i) broken left atrium (described above); ii) broken left ventricle, described as "blood is not flowing back out into the rest of the body", and iii) broken tricuspid valve, described using arrows as "blood is coming back up into this first part of the heart".

To assess recall of blood flow, we showed participants two screenshots of the heart from the video with arrows moving through each to represent the flow of blood. One set of arrows depicted the blood flowing in the correct way, while the other depicted the blood flowing in a simplified but incorrect way (see Figure 1). The arrows moved through each heart twice before the participant was allowed to respond to the question: "Which arrows show how blood really flows through the heart?" Both hearts were placed on colored backgrounds (i.e., purple and orange) to facilitate answering the question.

The four mechanistic questions were blocked together, with both the order of questions and order of response options and associated colors counterbalanced across participants.

Enjoyment of Learning Goal. To assess participants ' enjoyment of their learning goal condition, we asked, "Did you like your learning goal today? (Yes/No)" Based on their answer, they were then asked how much they liked or disliked their learning goal —a lot or just a little.

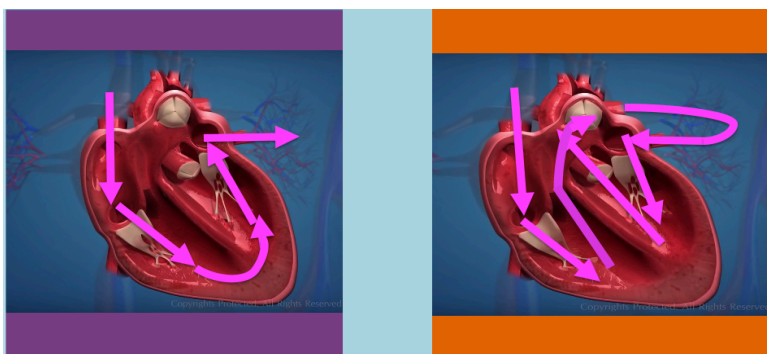

Figure 1: Static images depicting response options for the blood flow visualization question. Participants viewed the arrows moving through each heart, one at a time, and asked to report which heart shows how blood moves through the heart. Here, the purple heart depicts a simple but incorrect path of blood flow, while the orange depicts the relative counterintuitive but correct path of blood flow.

Procedure. Participants were told their learning goal and thereafter required to correctly identify it to ensure that they recalled the goal. Prior to watching the video, participants were asked if they wanted to watch the video about a cartoon heart "with cartoon blood moving around inside" to ensure that they were not squeamish about internal organs. After the brief distractor task, participants were reminded of their learning goal before the eight assessment questions. At the end of the task, participants were reminded again of their learning goal and reported their enjoyment of their learning 
goal. The whole task took an average of 15 minutes to complete.

Scoring. We computed overall performance on the assessment by averaging responses across all 8 questions. We also computed average performance across the two types of questions: labels and mechanism. One participant's response to one 'Broken Heart' question was omitted because their parent provided a response for their child before the child answered the question themselves.

Does a focus on mechanism enhance science learning? We predicted that children given a mechanistic learning goal would perform better both when assessing mechanistic and labels learning. In line with predictions, children given a mechanism-focused learning goal outperformed $(M=65 \%$, $S D=20 \%)$ children given a labels-focused learning goal $(M$ $=55 \%, S D=21 \% ; t(178)=3.28 ; p<.001)$.

To assess whether mechanistic learning goal boosted performance of each type of question, we also conducted two mixed-measures ANOVA (i.e., one for mechanism questions, one for labels questions) with question type as a withinsubjects variable and age and condition as between-subjects variables.

Labels. When assessing effects of condition and age on performance on labels questions, we found a marginal effect of condition: $F(1,174)=3.03, p=.08$, eta $a^{2}=.02$, with children given a mechanism learning goal performing marginally better $(M=59 \% ; S D=28 \%)$ than those given a labels learning goal $(M=52 \% ; S D=28 \%)$. Although it does not reach traditional levels of significance, it is noteworthy that children given a mechanistic goal marginally outperformed those in the other condition on the very questions that the labels-focused goal was targeted toward.

There was also a significant interaction between condition and individual question: $F(3,522)=2.81, p=.04$, eta ${ }^{2}=.02$. Children given a mechanistic learning goal performed significantly better on two of the four labels questions (i.e., number of chambers, labeling of right atrium; both Bonferroni $p$ 's <.05). There were no significant differences in performance on the other two labels questions (i.e., aorta, pulmonary artery).

We also found a main effect of age, with 6- to 7-year-olds recalling fewer labels $(M=43 \%, S D=27 \%)$ than both 8 - to 9 -year-olds $(M=60 \% ; S D=27 \%$; Bonferroni $p=.002)$ and 10 - to 11 -year-olds $(M=63 \% ; S D=27 \%$ Bonferroni $p<$ $.001)$. This suggests that older children are better able to retain terminology than younger children after a short lesson.

Mechanism. When assessing effects of condition and age on performance on mechanism questions, we found a significant main effect of condition: $F(1,173)=11.14, p=.001$, et $a^{2}=$ .06 , with children given a mechanism learning goal outperforming $(M=71 \% ; S D=23 \%)$ those given a labels learning goal $(M=58 ; S D=28 \%$. See Figure 2$)$. This effect did not interact with any other variables.
We also found a main effect of age, with 6- to 7-year-olds recalling fewer mechanisms $(M=56 \%, S D=25 \%)$ than both 8- to 9-year-olds $(M=67 \% ; S D=27 \%$; Bonferroni $p=.04)$ and 10- to 11-year-olds $(M=69 \%$; $S D=26 \%$; Bonferroni $p$ $=.02$. This suggests that older children are better able to retain mechanistic understandings than younger children after a short lesson.

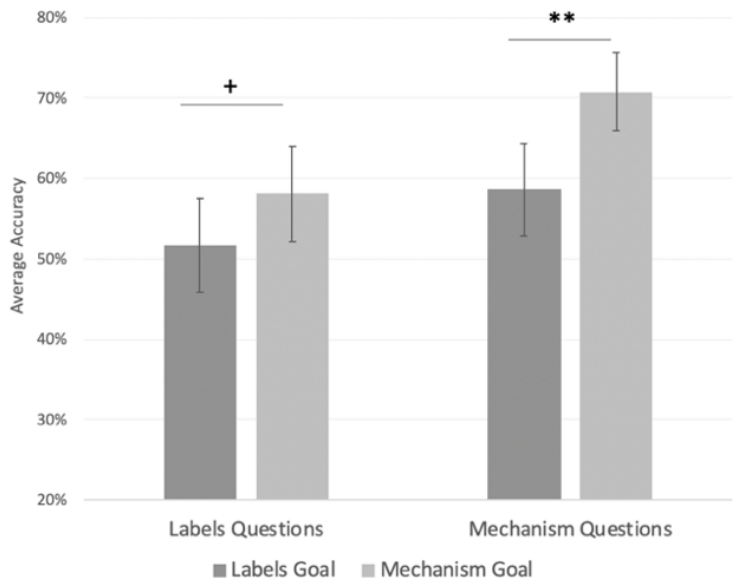

Figure 2: Effects of explicit learning goals on accuracy in knowledge assessments of both mechanism and labels of heart parts. Error bars display $95 \%$ confidence intervals. $* p<.05 ; * * *<.001$.

Summary. Thus, for both labels and mechanism questions, focusing on a mechanistic learning goal boosted children's performance. Surprisingly, for both question types, learning goal condition did not interact with age group. While older children learned more from the video overall, children of all ages benefitted similarly from a mechanistic learning goal, with enhanced learning of both mechanisms and labels.

Did children prefer mechanistic learning goals over labels learning goals? To investigate this question, we computed a Chi square test to investigate the between-subjects effect of learning goal condition (i.e., mechanistic v. labels goal) on reported enjoyment of learning goals. We did not have a strong prediction for this research question.

The Chi square was significant: $X^{2}(4,180)=11.41, p=.02$. Children who were given a mechanistic goal liked their learning goal more than those given a labels goal, as evidence by high frequency of reporting that they liked their goal " $a$ lot" (see Figure 3). In contrast, children who were given a labels learning goal more frequently said that they liked their goal "a little." Notably, the majority of children in both conditions most often reported liking their learning goals. Thus, although children in the mechanism condition enjoyed their learning goal more, the effects of goals on learning cannot be dismissed by concerns that children in the labels condition simply disliked their learning goal. 


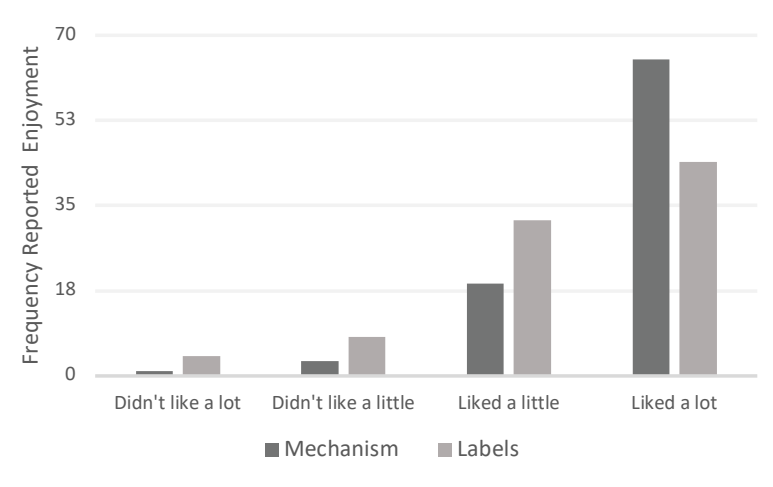

Figure 3: Differences in frequency of reported enjoyment of learning goals across conditions. Note: three participants were omitted from this graph (but included in Chi Square analyses) because they reported liking their learning goal 'in the middle' between 'a lot 'and 'a little.'

\section{General Discussion}

We investigated the effects of mechanistic learning goals on elementary children's learning and enjoyment during a video science lesson about the heart. Children ages 6 to 11 were given one of two learning goals: to either focus on mechanism (how the heart works) or to focus on labels (what the parts were called). We then measured children's recall of heart mechanism and labels taught in an educational video as well as their enjoyment of their learning goal. Research questions included: Does a focus on mechanism enhance science learning? and Do children prefer mechanistic learning goals over labels?

\section{A mechanistic focus enhances science learning}

Across sampled elementary-aged children, those who were given a mechanism-focused learning goal outperformed those who were given a labels-focused learning goal. Notably, this effect held for both types of question content. Thus, a mechanistic focus benefitted not only understanding of mechanism, but also understanding of labels.

A growing number of studies reveal that young children can understand mechanistic explanations (e.g., Metz, 1991; Haeusler \& Donovan, 2017). Moreover, mechanistic understandings may be critical to children's ability to generalize knowledge across domains (e.g., Russ et al., 2008;), and appear to benefit future learning (Schwartz et al., 2009; Kurkel et al., 2021). Our successful intervention reveals a way to promote such mechanistic thinking: through learning goals. Providing that the lessons feature mechanistic content, learning goals could be incorporated into elementary school science classrooms without adding the burden of additional course content preparation.

While it may be expected that a mechanistic learning goal should enhance learning of mechanistic content, it was particularly surprising that we saw the same benefits for recalling of the labels. This finding somewhat contradicts previous experiments on 3- to 6-year-olds wherein mechanistic explanations support learning of causal mechanisms, but not irrelevant perceptual details, such as the colors of parts (Legare \& Lombrozo, 2014). A key difference between our study and this previous finding is the superficiality of the non-causal details. Although we considered the names of parts to be a relatively superficial detail (i.e., compared to mechanisms), it is certainly not as superficial as the colors of parts. Indeed, the terminology should be considered more relevant to understanding than part color. Taken together, our results suggest that a focus on mechanism may help cohere non-causal details that are considered to be relevant to understanding the process (e.g., terminology but not incidental colors).

One reason why a mechanistic focus may promote learning _ including learning non-causal content - is because mechanisms provides causal continuity (e.g., Bogen \& Machamer, 2010) and thereby embedding context. Mechanistic explanations provide a sequential, story-like account of how each component part plays a role in an overarching system (Machamer, Darden \& Craver, 2000; Beuhner, 2012). The connections between interacting component parts may act as conceptual outlines to guide us in mentally retracing the steps of a mechanic sequence. In doing so, the mechanistic explanation may clarify causal connections within our mental models of the process (e.g., Vosniadou, 2002; Forbus \& Gentner 1997). Although the current research does not directly test this interpretation, the results align with this account. Future research should more directly investigate the role of contextual continuity in mechanistic learning and mental models.

In contrast to our findings, an increased focus on mechanism in elementary schools may be counterintuitive to adults. Adults tend to think that mechanistic content is too difficult for elementary school children to understand and prioritize more superficial content, such as labels, over mechanism (Betz, McCarthy \& Keil, under revision). Because of known benefits of mechanistic education and current K-12 science education guidelines (NGSS, 2013), these adult biases should be addressed so that they do not result in a de-emphasis of mechanistic content in the classroom.

\section{Children prefer mechanistic learning goals}

Children reported liking the mechanistic learning goal more than the labels learning goal. Although children in both conditions reported liking their learning goals, those who were given a mechanistic learning goal reported liking their goal "a lot" while those given a labels learning goal reported only liking their goal "a little." Thus, elementary school science enthusiasm may be bolstered by drawing student attention to mechanistic content, particularly in contrast to non-causal content (e.g., labels).

This finding reaffirms previous results indicating that children are drawn to mechanistic content and enjoy learning about causal mechanisms. A curiosity for mechanism is initially evident in preschool children who seek causal and explanatory information (e.g., Frazier, Gelman \& Wellman, 2009) and continues into adulthood (e.g., Ahn et al., 1995). 
Moreover, 9-year-olds became more interested in science after being taught mechanistic explanations about atomic theory (Haeusler \& Donovan, 2017). Given that adults and children alike value mechanistic explanations, and that children actively enjoy them, an increased emphasis on mechanism is warranted in early science education (Dickes et al., 2016; Kelemen, 2019).

Children may have enjoyed the mechanistic learning goals more because they link mechanistic content with learning. Indeed, children who were asked which book they would prefer to read in order to learn something were more likely to select a mechanistic book option over a book that simply teaches fun facts (Trouche et al., 2018) Moreover, children are less likely to seek additional information after they are given mechanistic explanations compared to more superficial ones (e.g., circular; Mills et al., 2019). Combined, these findings suggest that mechanistic explanations may be the ones that best satisfy children's drive to learn.

\section{Implications for Labels in Science Education}

Thus far, we have discussed science terminology as relatively superficial content with reduced long-term learning benefits compared to mechanistic explanations. This position largely stems from our belief that teaching labels can become problematic when it is overemphasized above other types of more explanatory content (e.g., Jones et al., 2004; Schmidt, Wang \& McKnight, 2005; Snow, 2008). However, we freely acknowledge that labels and terminology can be helpful and even necessary. For example, students need to learn some science vocabulary to be able to communicate their ideas to others (Henrichs et al., 2011). Moreover, shared labels can encourage children to notice relational similarities across items (Christie \& Gentner, 2014). Indeed, the labels in our task were introduced in ways that emphasize such commonalities across parts with common names (e.g., "the ventricles pump blood out of the heart"). We nevertheless saw a boost in performance on both types of questions when children were instructed to focus on the mechanism.

Thus, labels need not be the focus of science lessons. Our results show that children are able to pick up on labels, or other non-causal details, while focusing on other content within a lesson, such as mechanism. This finding echoes work with undergraduates who learn more about organic chemistry when they learn about the mechanisms involved before the details of the specifics of common chemical reactions (Flynn \& Ogilvie, 2015). By acknowledging and taking advantage of these benefits of focusing on in-depth mechanistic explanations, we can enhance the learning and enjoyment of STEM content while incidentally achieving other learning goals as well.

\section{Conclusion}

In line with recent educational standards, we sought to increase elementary students' attention to mechanisms through learning goals. Children who were explicitly told to focus on learning mechanistic content about a heart learned more about both causal mechanisms and non-causal labels more than those told to focus on learning labels. Notably, children given the mechanistic goal also reported more enjoyment of their learning goal. Thus, mechanistic learning goals can be used to enhance both scientific understanding and enjoyment of elementary school science lessons.

\section{Acknowledgments}

We thank all members of the Cognition and Development Laboratory for feedback on preliminary versions of this study, and Amanda M. McCarthy and Emory Richardson for their assistance with data collection. This research was funded by NSF \#DRL 1561143 granted to Frank Keil.

\section{References}

Ahn, W. K., Kalish, C. W., Medin, D. L., \& Gelman, S. A. (1995). The role of covariation versus mechanism information in causal attribution. Cognition, 54(3), 299352.

Bechtel, W., \& Abrahamsen, A. (2005). Explanation: A mechanist alternative. Studies in History and Philosophy of Science Part C: Studies in History and Philosophy of Biological and Biomedical Sciences, 36(2), 421-441.

Betz, N., McCarthy, A. M., \& Keil, F. (under revision). Minimizing Mechanism: Adult Perceptions of Mechanistic Content in Elementary School Science. CogSci

Bogen, J., \& Machamer, P. (2010). Mechanistic information and causal continuity.

Bonawitz, E., Shafto, P., Gweon, H., Goodman, N. D., Spelke, E., \& Schulz, L. (2011). The double-edged sword of pedagogy: Instruction limits spontaneous exploration and discovery. Cognition, 120(3), 322-330.

Buehner, M. J. (2012). Understanding the past, predicting the future: causation, not intentional action, is the root of temporal binding. Psychological science, 23(12), 14901497.

Christie, S., \& Gentner, D. (2014). Language helps children succeed on a classic analogy task. Cognitive science, 38(2), 383-397.

Chuey, A., McCarthy, A. M., Lockhart, K., Trouche, E., Sheskin, M., \& Keil, F. (under review). No Guts no Glory. Collins, A., \& Gentner, D. (1987). How people construct mental models. Cultural models in language and thought, 243, 243-265.

Coyne, M. D., McCoach, D. B., Loftus, S., Zipoli Jr, R., \& Kapp, S. (2009). Direct vocabulary instruction in kindergarten: Teaching for breadth versus depth. The Elementary School Journal, 110(1), 1-18.

Craik, K. J. W. (1952). The nature of explanation (Vol. 445). CUP Archive.

De Kleer, J., \& Brown, J. S. (1981). Mental models of physical mechanisms and their acquisition. Cognitive skills and their acquisition, 285-309.

Dickes, A. C., Sengupta, P., Farris, A. V., \& Basu, S. (2016). Development of mechanistic reasoning and multilevel explanations of ecology in third grade using agent-based models. Science Education, 100(4), 734-776. 
Flynn, A. B., \& Ogilvie, W. W. (2015). Mechanisms before reactions: A mechanistic approach to the organic chemistry curriculum based on patterns of electron flow. Journal of Chemical Education, 92(5), 803-810

Forbus, K., \& Gentner, D. (1997, June). Qualitative mental models: Simulations or memories. In Proceedings of the eleventh international workshop on qualitative reasoning (pp. 3-6).

Frazier, B. N., Gelman, S. A., \& Wellman, H. M. (2009). Preschoolers 'search for explanatory information within adult-child conversation. Child development, 80(6), 15921611.

Gentner, D., \& Stevens, A. L. (Eds.). (2014). Mental models. Psychology Press.

Haeusler, C., \& Donovan, J. (2020). Challenging the science curriculum paradigm: Teaching primary children atomicmolecular theory. Research in Science Education, 50(1), 23-52.

Henrichs, L. F., Leseman, P. P., Broekhof, K., \& De Lara, H. C. (2011). Kindergarten talk about science and technology. In Professional Development for Primary Teachers in Science and Technology (pp. 217-227). Brill Sense.

Jones, H., Black, B., Green, J., Langton, P., Rutherford, S., Scott, J., \& Brown, S. (2015). Indications of knowledge retention in the transition to higher education. Journal of Biological Education, 49(3), 261-273.

Jones, H. L., Green, J. R., Prendergast, J., \& Scott, J. (2018). Biology-specific vocabulary: students' understanding and lecturers' expectations of student understanding. Journal of Biological Education.

Keil, F. C., Levin, D. T., Richman, B. A., \& Gutheil, G. (1999). Mechanism and explanation in the development of biological thought: The case of disease. In D. L. Medin \& S. Atran (Eds.), Folkbiology (pp. 285-319). Cambridge, MA: MIT press

Kelemen, D. (2019). The magic of mechanism: Explanationbased instruction on counterintuitive concepts in early childhood. Perspectives on Psychological Science, 14(4), 510-522.

Kurkul, K. E., Castine, E., Leech, K., \& Corriveau, K. H. How does a switch work? The relation between adult mechanistic language and children's learning. Journal of Applied Developmental Psychology, 72, 101221.

Legare, C. H., \& Lombrozo, T. (2014). Selective effects of explanation on learning during early childhood. Journal of experimental child psychology, 126, 198-212.

Liquin, E. G., \& Lombrozo, T. (2020). Explanation-seeking curiosity in childhood. Current Opinion in Behavioral Sciences, 35, 14-20.

Lock, R. (1998). Advanced-level biology-is there a problem?. School Science Review, 80, 25-28.

Locke, E. A., Shaw, K. N., Saari, L. M., \& Latham, G. P. (1981). Goal setting and task performance: 1969-1980. Psychological bulletin, 90(1), 125.

Luce, M. R., \& Hsi, S. (2015). Science-relevant curiosity expression and interest in science: an exploratory study. Science Education, 99(1), 70-97.
Machamer, P., Darden, L., \& Craver, C. F. (2000). Thinking about mechanisms. Philosophy of science, 67(1), 1-25.

McNeil, N. M., \& Alibali, M. W. (2000). Learning mathematics from procedural instruction: Externally imposed goals influence what is learned. Journal of Educational Psychology, 92(4), 734.

Metz, K. E. (1985). The development of children's problem solving in a gears task: A problem space perspective. Cognitive Science, 9(4), 431-471.

Metz, K. E. (1991). Development of explanation: Incremental and fundamental change in children's physics knowledge. Journal of Research in Science Teaching, 28(9), 785-797.

Mills, C. M., Sands, K. R., Rowles, S. P., \& Campbell, I. L. (2019). "I Want to Know More!": Children Are Sensitive to Explanation Quality When Exploring New Information. Cognitive Science, 43(1), e12706.

Moray, N. (1998). Identifying mental models of complex human-machine systems. International Journal of Industrial Ergonomics, 22(4-5), 293-297.

NGSS Lead States. (2013a). Next Generation Science Standards: For states, by states (vol. 1, The Standards). Washington, DC: The National Academies Press. http://www.nextgenscience.org/next-generation-sciencestandards.

Russ, R. S., Scherr, R. E., Hammer, D., \& Mikeska, J. (2008). Recognizing mechanistic reasoning in student scientific inquiry: A framework for discourse analysis developed from philosophy of science. Science Education, 92(3), 499525.

Schunk, D. H., \& Rice, J. M. (1989). Learning goals and children's reading comprehension. Journal of Reading Behavior, 21(3), 279-293.

Schwartz, M. S., Sadler, P. M., Sonnert, G., \& Tai, R. H. (2009). Depth versus breadth: How content coverage in high school science courses relates to later success in college science coursework. Science education, 93(5), 798-826.

Sheskin, M., \& Keil, F. (2018). TheChildLab. com A video chat platform for developmental research.

Snow, C. (2008). Essay: What is the vocabulary of science. Teaching science to English language learners: Building on students 'strengths, 71-84.

Trouche, E., Chuey, A., Lockhart, K., \& Keil, F. (2017). Why Teach How Things Work? Tracking the Evolution of Children's Intuitions About Complexity. In CogSci.

Trouche, E., Chuey, A., Lockhart, K., \& Keil, F. (2018). Children Don't Just Wanna Have Fun: An Experimental Demonstration Of Children's Curiosity For How Things Work. In $\mathrm{CogS}$ Ci.

Vosniadou, S. (2002). Mental models in conceptual development. In Model-based reasoning (pp. 353-368). Springer, Boston, MA.

Vosniadou, S., \& Brewer, W. F. (1994). Mental models of the day/night cycle. Cognitive science, 18(1), 123-183. 